\title{
Signatures of noncommutative QED at photon colliders
}

\author{
Seungwon Baek, Dilip Kumar Ghosh, Xiao-Gang He, and W.-Y. P. Hwang \\ Department of Physics, National Taiwan University, Taipei 106, Taiwan
}

(Received 13 March 2001; published 13 July 2001)

\begin{abstract}
In this paper we study noncommutative (NC) quantum electrodynamics (QED) signatures at photon colliders through pair production of charged leptons $\left(l^{+} l^{-}\right)$and charged scalars $\left(H^{+} H^{-}\right)$. The $\mathrm{NC}$ corrections for the fermion pair production can be easily obtained since NC QED with fermions has been extensively studied in the literature. NC QED with scalars is less studied. To obtain the cross section for $\mathrm{H}^{+} \mathrm{H}^{-}$production, we first investigate the structure of NC QED with scalars, and then study the corrections due to the NC geometry to the ordinary QED cross sections. Finally by folding in the photon spectra for a $\gamma \gamma$ collider with laser backscattered photons from the $e^{+} e^{-}$machine, we obtain a 95\% C.L. lower bound on the NC scale using the above two processes. We find that, with $\sqrt{s}=0.5,1.0$, and $1.5 \mathrm{TeV}$ and integrated luminosity $L=500\left(\mathrm{fb}^{-1}\right)$, the $\mathrm{NC}$ scale up to $0.7,1.2$, and $1.6 \mathrm{TeV}$ can be probed, respectively, while, for monochromatic photon beams, these numbers become 1.1, 1.7, $2.6 \mathrm{TeV}$, respectively.
\end{abstract}

DOI: 10.1103/PhysRevD.64.056001

PACS number(s): 11.15.-q, 11.25.Mj, 13.40.-f

\section{INTRODUCTION}

The property of space-time has fundamental importance in understanding the laws of nature. Noncommutative (NC) quantum field theory provides an alternative to ordinary quantum field theory which may shed some light on the detailed structure of space-time and has been studied in the past [1]. Recently NC quantum field theory and its applications have also been developed within string theories where it arises in low energy excitations of D-branes in the presence of a certain $U(1)$ background field and has received a lot of attention [2]. A simple way to modify the commutation relation for ordinary space-time $x$ is defined, with the modified space-time coordinate $\hat{X}$, as

$$
\left[\hat{X}_{\mu}, \hat{X}_{\nu}\right]=i \theta_{\mu \nu}=\frac{i}{\Lambda^{2}} c_{\mu \nu} .
$$

In the above the parameter $\Lambda$, which has the dimension of energy, signifies the scale where $\mathrm{NC}$ effects become relevant. $c_{\mu \nu}$ is a real antisymmetric matrix with elements of order one which commute with the space-time coordinate $x^{\mu}$.

Phenomenologically the NC scale $\Lambda$ can take any value, the likely one being on the order of the Planck scale $\mathrm{M}_{\mathrm{Pl}}$. However, the recent studies in the area of large extra dimensions show that gravity becomes strong at the TeV scale [3], and also one might see some stringy effects at this scale. Hence, it is justified if one takes the scale of $\Lambda$ to be on the order of the $\mathrm{TeV}$ scale. If this is the case, then whether $\mathrm{NC}$ geometry has anything to do with reality has to be tested experimentally. In this context the Next Generation $e^{+} e^{-}$ Linear Collider (NLC) will be an ideal machine to probe such new physics effects. The $e^{+} e^{-}$version of NLC can be modified to give $e^{-} e^{-}, e \gamma$, and $\gamma \gamma$ modes of the collider. Some of the authors have already studied the NC effects at NLC [4-6].

In this paper we study signatures of NC quantum electrodynamics (QED) at $\gamma \gamma$ colliders. $\gamma \gamma$ colliders can be very sensitive to certain new physics beyond the standard model [7]. We also find that $\gamma \gamma$ colliders can provide interesting information about the scale $\Lambda$ of noncommutative geometry. Two processes $\gamma \gamma \rightarrow l^{+} l^{-}$and $\gamma \gamma \rightarrow H^{+} H^{-}$will be studied in detail. These processes are particularly interesting in studying the noncommutative QED effects because at leading order they are purely QED processes, eliminating problems associated with difficulties in having a full gauge theory for $S U(3)_{C} \times S U(2)_{L} \times U(1)_{Y}$. This is because that only the $\mathrm{U}(N)$ group can be gauged consistently with NC geometry [8]. The gauge group of the standard model has to be enlarged in the presence of NC geometry, Eq. (1). If weak interaction is involved, then there is a problem in identifying $\mathrm{NC}$ effects such as the process $e^{-} e^{-} \rightarrow e^{-} e^{-}$where exchange of the $Z$ boson also contributes [5].

The paper is organized as follows. In Sec. II we study the $\gamma \gamma \rightarrow l^{+} l^{-}$process in NC QED with monochromatic photon beams and laser backscattered photon beams for three values of center-of-mass energies $0.5,1$, and $1.5 \mathrm{TeV}$ [9]. We obtain 95\% C.L. lower bound which can be probed on the NC scale $\Lambda$. In Sec. III we study the noncommutative scalar QED. We first derive the corresponding Feynman rules and use them to obtain 95\% C.L. lower bound on $\Lambda$ from the $\gamma \gamma \rightarrow H^{+} H^{-}$ process. Finally in Sec. IV, we summarize our results.

\section{II. $\gamma \gamma \rightarrow l^{+} l^{-}$IN NC QED}

In this section we study the effects of NC QED in the $\gamma \gamma \rightarrow l^{+} l^{-}$process. NC QED with fermions has been studied extensively [4]. The relevant Feynman rules are shown in Fig. 1 and the Feynman diagrams for $\gamma \gamma \rightarrow l^{+} l^{-}$are shown in Fig. 2. It is clear from the Feynman rules as well as from Fig. 2 that there are extra contributions to the ordinary QED. The ordinary QED vertex is modified to have a momentum dependent phase factor. Apart from this there are completely new triple and quartic photon vertices making the NC QED like a non-Abelian gauge theory. The origin of phase factors in the vertices can be traced back to the famous Weyl-Moyal correspondence [10] which we will state later. These new contributions to the existing vertices result in deviations from the ordinary QED predictions. We obtain the unpolar- 


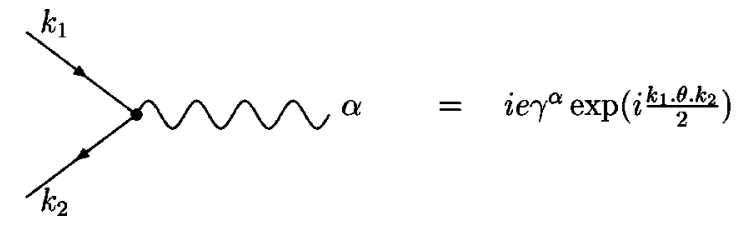

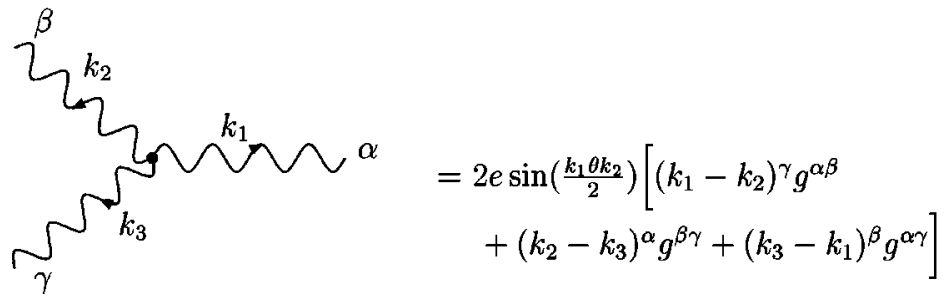

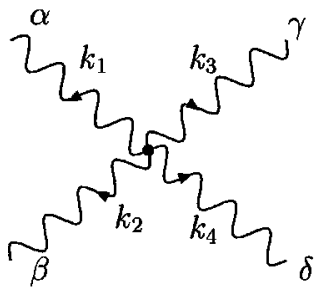

FIG. 1. Feynman rules for NC QED with fermions.

$$
\begin{gathered}
=4 i e^{2}\left[\left(g^{\alpha \delta} g^{\beta \gamma}-g^{\alpha \gamma} g^{\beta \delta}\right) \sin \left(\frac{k_{1} \theta k_{2}}{2}\right) \sin \left(\frac{k_{3} \theta k_{4}}{2}\right)\right. \\
+\left(g^{\alpha \gamma} g^{\beta \delta}-g^{\alpha \beta} g^{\delta \gamma}\right) \sin \left(\frac{k_{3} \theta k_{1}}{2}\right) \sin \left(\frac{k_{2} \theta k_{4}}{2}\right) \\
\left.+\left(g^{\alpha \beta} g^{\gamma \delta}-g^{\alpha \delta} g^{\beta \gamma}\right) \sin \left(\frac{k_{1} \theta k_{4}}{2}\right) \sin \left(\frac{k_{2} \theta k_{3}}{2}\right)\right]
\end{gathered}
$$

ized differential cross section for the $\gamma\left(k_{1}\right) \gamma\left(k_{2}\right)$ $\rightarrow l^{-}\left(p_{1}\right) l^{+}\left(p_{2}\right)$ process in the massless limit as

$$
\frac{d \sigma}{d z d \phi}=\frac{\alpha^{2}}{2 \hat{s}}\left[\frac{\hat{u}}{\hat{t}}+\frac{\hat{t}}{\hat{u}}-4 \frac{\hat{t}^{2}+\hat{u}^{2}}{s^{2}} \sin ^{2} \delta\right],
$$

where the NC phase is $\delta=\left(k_{1} \cdot \theta \cdot k_{2} / 2\right) . \hat{s}=\left(k_{1}+k_{2}\right)^{2}=\left(p_{1}\right.$ $\left.+p_{2}\right)^{2}, \hat{t}=\left(k_{1}-p_{1}\right)^{2}=\left(k_{2}-p_{2}\right)^{2}$, and $\hat{u}=\left(k_{1}-p_{2}\right)^{2}=\left(k_{2}\right.$ $\left.-p_{1}\right)^{2}$ are the standard Mandelstam variables. In the $\gamma \gamma$ center-of-mass frame, $\hat{t}$ and $\hat{u}$ can be further written in terms of $\hat{s}$ and the angle $\hat{\theta}$ between $\vec{k}_{1}$ (the $z$ direction) and $\vec{p}_{1}$ with $\hat{t}=-(\hat{s} / 2)(1-z), \hat{u}=-(\hat{s} / 2)(1+z)$, where $z=\cos \hat{\theta}$. The angle $\phi$ is the azimuthal angle. So, the NC effect in the $\gamma \gamma \rightarrow l^{+} l^{-}$process lies in the even function $\sin ^{2} \delta$ of $\delta$ and one can recover the ordinary QED result by taking the limit $\delta \rightarrow 0$. The phase $\delta$ arises from the $s$-channel triple photon vertex diagram and also from the interference between the $s t$-channel and $s u$-channel diagrams.

The cross sections are only sensitive to the NC parameter $c_{0 z}$ because the corrections only depend on $\sin ^{2}\left(\frac{1}{2} k_{1} \cdot \theta \cdot k_{2}\right)$ which is equal to $\sin ^{2}\left[(\hat{s} / 4)\left(c_{0 z} / \Lambda^{2}\right)\right]$ for head-on photon collisions. Because of this the cross section does not depend on the azimuthal angle $\phi$ and will be integrated over in the

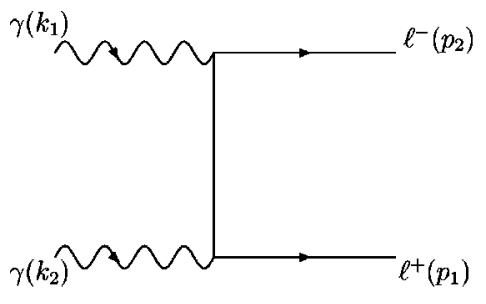

(a)

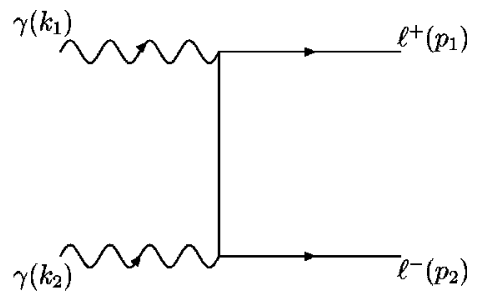

(b)
FIG. 2. Feynman diagram for $\gamma \gamma \rightarrow l^{+} l^{-}$in the presence of NC QED. 


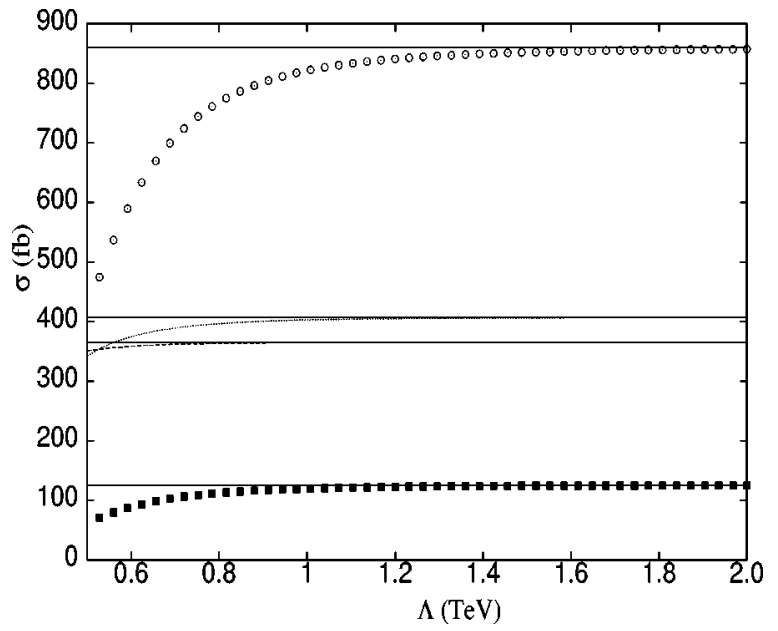

FIG. 3. Variation of $\sigma\left(\gamma \gamma \rightarrow l^{+} l^{-}\right)$and $\sigma\left(\gamma \gamma \rightarrow H^{+} H^{-}\right)$with the NC scale $\Lambda$ at the $\sqrt{s}_{e^{+} e^{-}}=1 \mathrm{TeV}$ NLC machine. The notations are as follows: (i) $\sigma\left(\gamma \gamma \rightarrow l^{+} l^{-}\right)$with monochromatic and with laser backscattered photon beams are represented by the curve with $\odot$ and with dotted lines, respectively. The solid lines adjacent to these correspond to commutative QED contribution. (ii) $\sigma(\gamma \gamma$ $\rightarrow H^{+} H^{-}$) with monochromatic and with laser backscattered photon beams are represented by the curve with dark boxes and with dashed lines, respectively. The solid lines adjacent to these correspond to ordinary QED contribution. For this we have fixed the scalar mass $m_{H}=100 \mathrm{GeV}$.

cross section from now on. In our later discussions, we will set $c_{0 z}=1$ and study the sensitivity to the NC scale $\Lambda$.

$\mathrm{NC}$ theories violate Lorentz invariance. In general in different frames, the parameter $c_{0 z} / \Lambda^{2}$ may be different. However, in cases with head-on photon collisions $k_{1} \cdot \theta \cdot k_{2}$ $=\left(c_{0 z} / \Lambda^{2}\right)\left(k_{1}^{0} k_{2}^{z}-k_{1}^{z} k_{2}^{0}\right)$. Because of the fact that $\left(k_{1}^{0} k_{2}^{z}\right.$ $\left.-k_{1}^{z} k_{2}^{0}\right)$ is invariant under a boost along the $z$ direction, the $c_{0 z} / \Lambda^{2}$ is the same in all frames boosted in the $z$ direction. This is important for our later discussions on constraining the parameter $c_{0 z} / \Lambda^{2}$ using backscattered photon beams, which involves boosting along the $z$ direction from the center of mass frame. Although the frames may be different for each energy of photons, we obtain constraint on the same parameter $c_{0 z} / \Lambda^{2}$. This is also true for our later discussions on charged scalar production. One should note that the above situation does not happen in general. For example, in the $\gamma\left(k_{1}\right) \gamma\left(k_{2}\right) \rightarrow \gamma\left(k_{3}\right) \gamma\left(k_{4}\right)$ process, because several combinations of $k_{i} \cdot \theta \cdot k_{j}$ are involved [5], a boost along one direction cannot make all of them invariant.

Now we study the cross section as a function of the NC scale $\Lambda$. In obtaining the cross section we sum over three leptonic $(e, \mu, \tau)$ generations. We also assume the identification efficiencies for $e$ and $\mu$, and $\tau$ to be $100 \%$ and $60 \%$, respectively. One should note that, due to the neglect of small lepton masses, there are singularities in the cross section when $z= \pm 1$. To avoid these singularities, we demand that the rapidity $\left|\eta_{l}\right|$ of each lepton should be less than 1 . This choice of rapidity cut corresponds to an angular cut $|z|<0.76$ on each lepton. In Fig. 3 we plot the variation of this cross section with $\Lambda$ for a monochromatic (line with $\odot$ ) photon collider with energy $\sqrt{s}_{\gamma \gamma}=1 \mathrm{TeV}$. The adjacent solid

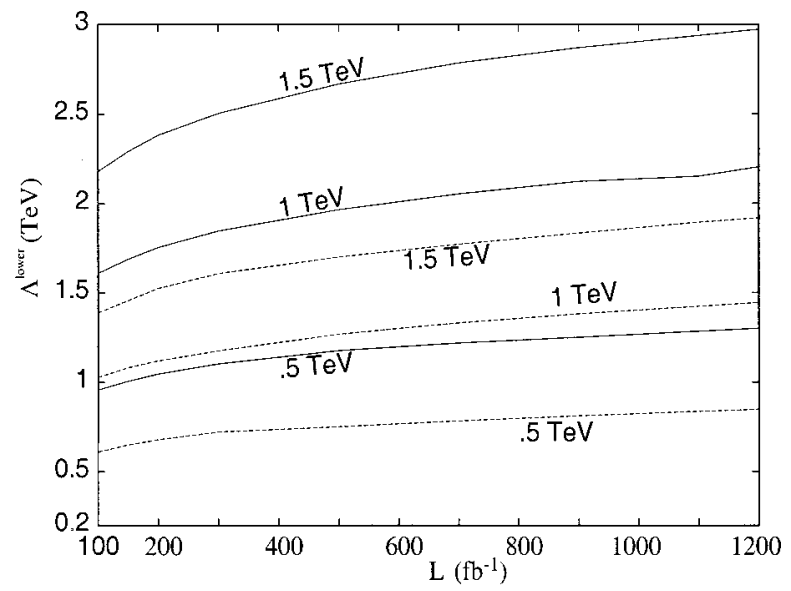

FIG. 4. 95\% C.L. lower bound on $\Lambda$ can be probed as a function of integrated luminosity from the $\gamma \gamma \rightarrow l^{+} l^{-}$process. The solid lines are using monochromatic photon beams, while the dashed lines are with backscattered photons. The numbers adjacent to each curve represent the $\sqrt{s}_{e^{+} e^{-}}$.

line represents the ordinary QED cross section. It can be seen that the ordinary QED gets a negative contribution from NC QED, and as the NC scale $\Lambda$ increases the NC QED result asymptotically approaches the ordinary QED one.

To study the possible sensitivity of NLC to the NC scale $\Lambda$ we perform a $\chi^{2}(\Lambda)$ fit assuming that statistical errors are Gaussian and that there are no systematic errors. $\chi^{2}(\Lambda)$ is given by

$$
\chi^{2}=L \frac{\left(\sigma_{\mathrm{NC}}(\Lambda)-\sigma_{\mathrm{SM}}\right)^{2}}{\sigma_{\mathrm{SM}}},
$$

where $L$ is the integrated luminosity, $\sigma_{\mathrm{SM}}$ is the ordinary QED total cross section, and $\sigma_{\mathrm{NC}}(\Lambda)$ is the NC QED cross section. By demanding $\chi^{2} \geqslant 4$, we obtain the lower bound on the NC scale $\Lambda$ at $95 \%$ C.L. We denote this bound by $\Lambda^{\text {lower }}$. We take three machine energies $\sqrt{s}_{e^{+} e^{-}}=0.5,1.0$, and 1.5 $\mathrm{TeV}$, for illustrations. In the case of the monochromatic photon collider, $\sqrt{s}_{\gamma \gamma}=\sqrt{s}_{e^{+}} e^{-}$.

In Fig. 4 we show the scale $\Lambda^{\text {lower }}$ as a function of $L$ from the $\gamma \gamma \rightarrow l^{+} l^{-}$process. The solid lines in Fig. 4 are represented as a function of integrated luminosity $L$ for the monochromatic photon beams. The higher the $\sqrt{s}_{e^{+}} e^{-}$the larger the value of $\Lambda$ can be probed for a fixed integrated luminosity. This behavior can be understood from the nature of the NC correction term which goes as $\sim s^{2} / \Lambda^{4}$ to this process.

Until now, we have discussed the monochromatic photon beams. However, it is very difficult to obtain such a beam in practice. A realistic method of obtaining the high energy photon beam is to use the laser backscattering technique on an electron or positron beam, which produces abundant hard photons along nearly the same direction as the original electron or positron beam. The photon beam energy obtained in 
this way is not monochromatic. The energy spectrum of the backscattered photon is given by [11]

$$
\begin{aligned}
& f(x)=\frac{1}{D(\xi)}\left[1-x+\frac{1}{1-x}-\frac{4 x}{\xi(1-\xi)}+\frac{4 x^{2}}{\xi^{2}(1-x)^{2}}\right] \\
& D(\xi)=\left(1-\frac{4}{\xi}-\frac{8}{\xi^{2}}\right) \ln (1+\xi)+\frac{1}{2}+\frac{8}{\xi}-\frac{1}{2(1+\xi)^{2}},
\end{aligned}
$$

where $x$ is the fraction of the energy of the incident $e^{ \pm}$beam. The parameter $\xi$ is determined to be $2(1+\sqrt{2})$ by requiring that the backscattered photon have the largest possible energy, but does not interfere with the incident photon to create an unwanted $e^{+} e^{-}$pair which sets $x_{\max }=\xi /(1+\xi) \approx 0.828$. The cross section at such a $\gamma \gamma$ collider with the $e^{+} e^{-}$collider center of mass frame energy $\sqrt{s}$ is given by

$$
\sigma=\int_{x_{1 \min }}^{x_{\max }} d x_{1} f\left(x_{1}\right) \int_{x_{2 \min }}^{x_{\max }} d x_{2} f\left(x_{2}\right) \int_{z_{\min }}^{z_{\max }} d z \frac{d \sigma\left(x_{1} x_{2} s, z\right)}{d z} .
$$

To avoid the singularities at $z= \pm 1$ in $\gamma \gamma \rightarrow l^{+} l^{-}$, we make a cut on the rapidity of each lepton in the laboratory frame to be less than 1 and also a cut on the lepton energy such that the minimal values for $x_{1,2}$ to be $x_{1,2 \min }=0.5$. With this choice of cuts we show the variation of cross section with $\Lambda$ in Fig. 3. The dotted lines represent the NC QED cross section, while the adjacent solid lines correspond to the ordinary QED results. Compared to the monochromatic case, the cross section decreases. Naively one would expect it to be the other way around because the cross section decreases with energy. However, because of the cut on $x_{1,2 \min }$, a certain portion of the scattering is also cut off, which results in a smaller cross section.

In Fig. 4 we present $\Lambda^{\text {lower }}$ as a function of $L$ (dashed line). In this case, for a given $\sqrt{s}$ and integrated luminosity, the 95\% C.L. lower bounds are weaker than that of monochromatic photons. For example, at $\sqrt{s}_{e^{+} e^{-}}=1 \mathrm{TeV}$ and assuming the integrated luminosity $L=500 \mathrm{fb}^{-1}$ the $95 \%$ C.L. lower bound can be probed on $\Lambda$ is $1.2 \mathrm{TeV}$, while in the monochromatic case, the corresponding bound is 1.6 $\mathrm{TeV}$. This is due to the fact that the available $\gamma \gamma$ center-ofmass energy is not fixed but has an energy spectrum, which suppresses the NC effect.

\section{III. $\gamma \gamma \rightarrow H^{+} H^{-}$IN NC SCALAR QED}

NC QED with scalars are less studied. In order to study $\gamma \gamma \rightarrow H^{+} H^{-}$, we first construct the NC QED Lagrangian with scalars in the following. The Lagrangian in the ordinary quantum field theory relevant to $\gamma \gamma \rightarrow H^{+} H^{-}$is given by

$$
L=\left(D^{\mu} H^{-}\right) *\left(D_{\mu} H^{-}\right)-m_{H}^{2} H^{+} H^{-},
$$

where $D_{\mu}=\partial_{\mu}-i e A_{\mu}$ is the covariant derivative.

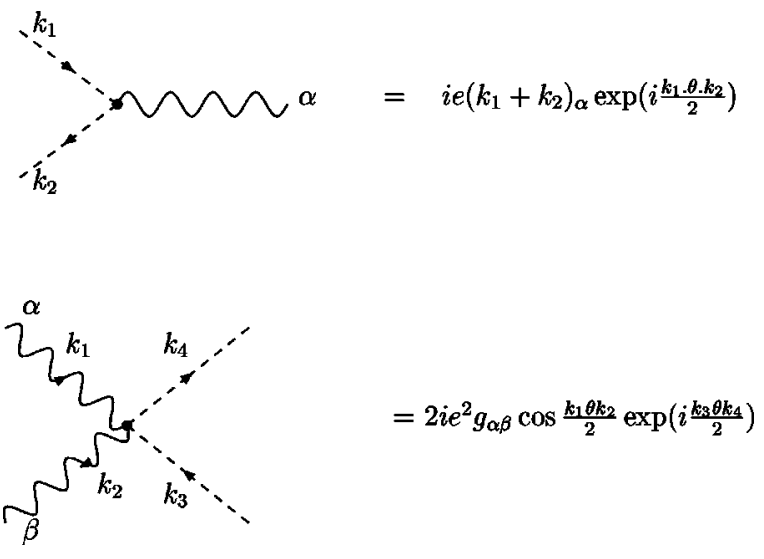

FIG. 5. Feynman rules for NC scalar QED.

When the above Lagrangian is formulated with noncommutative coordinates, there are corrections. NC quantum field theory can be easily studied using the Weyl-Moyal correspondence replacing the product of two fields $A(\hat{X})$ and $B(\hat{X})$ with NC coordinates by [10]

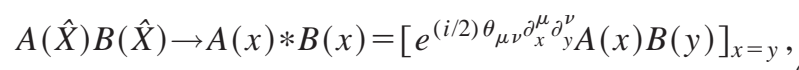

where $x$ and $y$ are the ordinary coordinates, and $\partial_{x}=\partial / \partial x$, $\partial_{y}=\partial / \partial y$.

Under an infinitesimal local gauge transformation $\lambda(x)$, the transformation law for $H$ is given by

$$
\begin{aligned}
\operatorname{Re} H^{-}(x) \rightarrow & \operatorname{Re} H^{-}(x)-\left.\cos \left(\frac{1}{2} \theta_{\mu \nu} \partial_{x}^{\mu} \partial_{y}^{\nu}\right) \lambda(x) \operatorname{Im} H^{-}(y)\right|_{x=y} \\
& -\left.\sin \left(\frac{1}{2} \theta_{\mu \nu} \partial_{x}^{\mu} \partial_{y}^{\nu}\right) \lambda(x) \operatorname{Re} H^{-}(y)\right|_{x=y}
\end{aligned}
$$

$\operatorname{Im} H^{-}(x) \rightarrow \operatorname{Im} H^{-}(x)$

$$
\begin{aligned}
& +\left.\cos \left(\frac{1}{2} \theta_{\mu \nu} \partial_{x}^{\mu} \partial_{y}^{\nu}\right) \lambda(x) \operatorname{Im} H^{-}(y)\right|_{x=y} \\
& -\left.\sin \left(\frac{1}{2} \theta_{\mu \nu} \partial_{x}^{\mu} \partial_{y}^{\nu}\right) \lambda(x) \operatorname{Re} H^{-}(y)\right|_{x=y} .
\end{aligned}
$$

Writing the Lagrangian in NC geometry, one obtains the tree level NC QED with scalars. We have

$$
\begin{aligned}
L= & \left(\partial_{\mu} H^{+}+i e H^{+} * A_{\mu}\right) *\left(\partial^{\mu} H^{-}-i e A^{\mu} * H^{-}\right) \\
& -m_{H}^{2} H^{+} * H^{-} .
\end{aligned}
$$

Because of the NC properties, the ordering of the fields in the above equation is important and should not be misplaced. From this Lagrangian one obtains the Feynman rules given in Fig. 5. The structure shows some similar momentum dependent phase factor as in the NC QED with fermions. The Feynman diagrams for $\gamma \gamma \rightarrow H^{+} H^{-}$are shown in Fig. 6. In this case we also get an additional contribution to the normal 


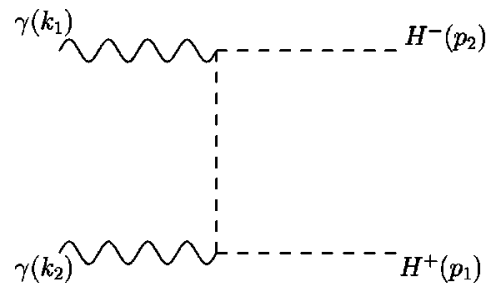

$(a)$

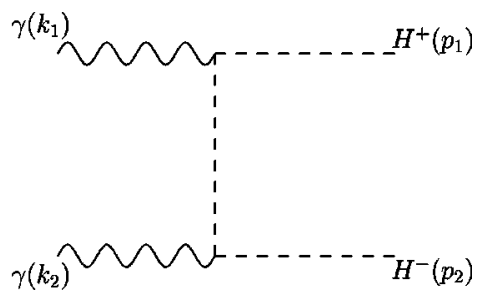

(b)

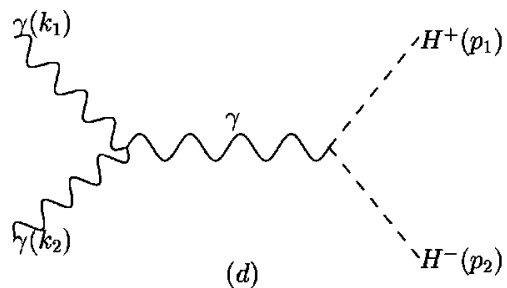

FIG. 6. Feynman diagram for $\gamma \gamma \rightarrow H^{+} H^{-}$in the presence of NC QED.

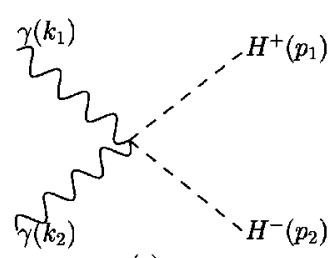

(c)

$$
\begin{aligned}
\frac{d \sigma}{d z d \phi}= & \frac{\alpha^{2} \hat{\beta}}{4 \hat{s}}\left[\frac{\left(m_{H}^{2}+\hat{t}\right)^{2}}{\left(m_{H}^{2}-\hat{t}\right)^{2}}+\frac{\left(m_{H}^{2}+\hat{u}\right)^{2}}{\left(m_{H}^{2}-\hat{u}\right)^{2}}+\frac{8 m_{H}^{4}}{\left(m_{H}^{2}-\hat{t}\right)\left(m_{H}^{2}-\hat{u}\right)}\right] \\
& \times\left[1-4 \frac{\left(m_{H}^{2}-\hat{t}\right)\left(m_{H}^{2}-\hat{u}\right)}{\hat{s}^{2}} \sin ^{2} \delta\right]
\end{aligned}
$$

where the $\mathrm{NC}$ phase $\delta$ has been defined earlier, $\hat{t}$ $=m_{H}^{2}-(\hat{s} / 2)(1-\hat{\beta} z), \quad \hat{u}=m_{H}^{2}-(\hat{s} / 2)(1+\hat{\beta} z), \quad$ and $\hat{\beta}$ $=\sqrt{1-4 m_{H}^{2} / \hat{s}}$ is the velocity of the charged scalar. In this case also, in the limit $\delta \rightarrow 0$, one obtains the pure QED result. Again this process depends only on $c_{0 z} / \Lambda$.

The scalars are similar to charged Higgs bosons in multiHiggs-boson models. However the decay products are not clear because the minimal standard model for electroweak interactions has to be extended with NC geometry. The charged scalar decay products may be modified. We will assume that the decay products of $H$ are similar to the charged Higgs scalars in multi-Higgs models and can be studied experimentally. One may also formulate NC QED with composite charged scalars, such as $\pi^{ \pm}$and $K^{ \pm}$, which will be commented on later.

The variation of unpolarized cross sections with $\Lambda$ for scalar mass $m_{H}=100 \mathrm{GeV}$ at $\sqrt{s}_{\gamma \gamma}=1 \mathrm{TeV}$ is also shown in Fig. 3 for both monochromatic (line with dark boxes) and laser backscattered (dashed lines) photon beams. The corresponding ordinary QED contributions are also depicted by the solid lines. From this figure it can be seen that the ordinary QED gets a negative contribution from NC QED like the $\gamma \gamma \rightarrow l^{+} l^{-}$process, and as the NC scale $\Lambda$ increases the NC QED contribution asymptotically approaches the ordinary QED result. It is interesting to note that the cross section in the backscattered case is larger than that of the monochromatic case, unlike the $\gamma \gamma \rightarrow l^{+} l^{-}$case discussed earlier. This is because in this case no cut on the final product energy is applied, therefore all contributions are included. However, the monochromatic case still has larger deviation between the ordinary and noncommutative QED as can be seen in Fig. 3. where $n$ is any arbitrary four-vector and $k$ is the photon fourmomentum. The same technique has been applied in the above charged lepton pair production study. The unpolarized differential cross section in the $\gamma \gamma$ center-of-mass frame is given by 

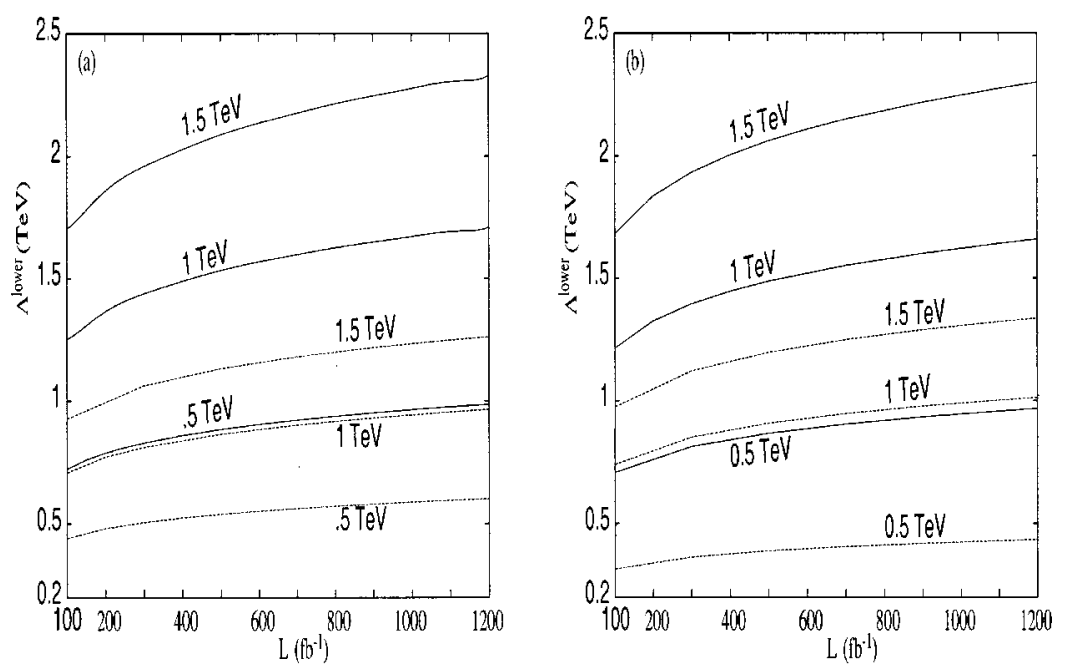

FIG. 7. $95 \%$ C.L. lower bound on $\Lambda$ can be probed as a function of integrated luminosity from the $\gamma \gamma \rightarrow H^{+} H^{-}$process for $m_{H}=100 \mathrm{GeV}$ (a) and $200 \mathrm{GeV}$ (b). The solid lines use monochromatic photon beams, while the dashed lines use backscattered photons. The numbers adjacent to each curve represent the $\sqrt{s_{e^{+}} e^{-}}$.

Now we will discuss our results on $\Lambda^{\text {lower }}$ for $\gamma \gamma$ $\rightarrow H^{+} H^{-}$with monochromatic photon beams. We use two charged scalar masses, $m_{H}=100$ and $200 \mathrm{GeV}$, for illustrations. We display $\Lambda^{\text {lower }}$ as a function of $L$ by the solid lines in Fig. 7(a) $\left(m_{H}=100 \mathrm{GeV}\right)$ and Fig. $7(\mathrm{~b})\left(m_{H}=200 \mathrm{GeV}\right)$. The numbers adjacent to each curve correspond to the monochromatic photon collider. It is clear from these two figures that $\Lambda^{\text {lower }}$ depends on the scalar mass. The lighter the mass, the larger the scale one can explore for a given $\sqrt{s}$ and integrated luminosity. For example, with $\sqrt{s}_{\gamma \gamma}=1 \mathrm{TeV}, \Lambda^{\text {lower }}$ are $1.53 \mathrm{TeV}$ for $m_{H}=100 \mathrm{GeV}$ and $1.48 \mathrm{TeV}$ for $m_{H}=200$ $\mathrm{GeV}$, respectively. Like the dilepton final state, here one can also probe the larger value of $\Lambda$ if one goes to higher energies.

The results for the laser backscattered photon beams are also shown in Figs. 7(a) and 7(b) by the dotted lines. In this case, there are no singularities at $z= \pm 1$. Therefore we will let $z$ vary within the full allowed range, that is with $z_{\min }=$ $-1, z_{\max }=1$. The integration lower limits for $x_{1}$ and $x_{2}$ are $x_{1 \min }=4 m_{H}^{2} / s x_{\max }$ and $x_{2 \min }=4 m_{H}^{2} / s x_{1}$. The maximum values of $x_{1}$ and $x_{2}$ have already been mentioned in Sec. II. Using Eq. (3) we then obtain $\Lambda^{\text {lower }}$ as a function of integrated luminosity $L$, which is shown by the dotted lines in Fig. 7. As before, we also study this case for two values of scalar masses 100 and $200 \mathrm{GeV}$. We see that the bounds which can be probed on the scale are in the range of $0.8-1.2$, $0.7-1.0$, and $0.4-0.6 \mathrm{TeV}$ for $\sqrt{s}_{\gamma \gamma}=1.5,1.0$, and $0.5 \mathrm{TeV}$, respectively. These bounds are slightly lower than that obtained in $\gamma \gamma \rightarrow l^{+} l^{-}$.

If the theory is applicable to composite particles such as $\pi^{ \pm}$and $K^{ \pm}$, the NC scale that can be probed with $L$ $=500 \mathrm{fb}^{-1}$ is $1.5 \mathrm{TeV}(1.2 \mathrm{TeV})$ for monochromatic (backscattered) photon beams for $\sqrt{s}_{\gamma \gamma}=1 \mathrm{TeV}\left(\sqrt{s}_{e^{+} e^{-}}=1 \mathrm{TeV}\right)$. Of course it may be difficult to carry out such experiments with energies as high as what we are considering.

In the above discussions we have used tree level cross sections, especially our reference ordinary cross sections $\sigma_{\mathrm{SM}}$. There are loop contributions which may lead to the change of $\chi^{2}$ compared with when tree cross sections are used. However, the loop corrections are much smaller than the NC corrections for $\chi^{2}$ as large as 4 . The bounds we obtained are for NC corrections to good approximations.

\section{CONCLUSIONS}

In summary, we have examined the feasibility of observing the experimental signature of noncommutative QED by studying the dilepton and a pair of charged scalar productions at a high energy photon collider. We have parametrized the effect of NC QED by an antisymmetric matrix $c_{\mu \nu}$ and an overall NC scale $\Lambda$. We found that in our processes only $c_{0 z}$ contributes. Throughout our analysis we have set this parameter to 1 and studied the sensitivities of $\gamma \gamma \rightarrow l^{+} l^{-}$and $\gamma \gamma \rightarrow H^{+} H^{-}$processes on the NC scale $\Lambda$.

We first studied the sensitivity for monochromatic $\gamma \gamma$ colliders. The variations of $\sigma\left(\gamma \gamma \rightarrow l^{+} l^{-}\right)$and $\sigma(\gamma \gamma$ $\rightarrow H^{+} H^{-}$) with the NC scale $\Lambda$ at $\sqrt{s}_{\gamma \gamma}=1 \mathrm{TeV}$ were obtained. We found that there are visible deviations between ordinary and NC QED predictions for small $\Lambda$, but when $\Lambda$ becomes larger, $\sigma_{\mathrm{NC}}$ asymptotically approaches $\sigma_{\mathrm{SM}}$. We also obtained a $95 \%$ C.L. lower limit which can be probed on $\Lambda$ from the above mentioned two processes as functions of the integrated luminosity $L$. It turned out that the higher the available center-of-mass energy, the larger the NC scale $\Lambda$ one can probe. We found that with $\sqrt{s}_{\gamma \gamma}=0.5,1.0$, and 1.5 TeV and integrated luminosity $L=500\left(\mathrm{fb}^{-1}\right)$ the $\mathrm{NC}$ scales can be probed up to $1.1,1.7$, and $2.6 \mathrm{TeV}$, respectively.

Next we considered a more realistic case, where, the photon beams are obtained by a laser backscattered from $e^{ \pm}$ beams. In this case, the available $\gamma \gamma$ center-of-mass energy has a spectrum with a maximum energy around $80 \%$ of the $\sqrt{s}_{e^{+}} e^{-}$. In general bounds on the scale that can be probed become lower. We have observed that for $\sqrt{s}_{e^{+} e^{-}}=0.5,1.0$, and $1.5 \mathrm{TeV}$, with the integrated luminosity $L=500\left(\mathrm{fb}^{-1}\right)$, the NC scales up to $0.7,1.2$, and $1.6 \mathrm{TeV}$, respectively, can be probed.

In both monochromatic and laser backscattered photon collider cases, the bounds on $\Lambda$ that can be probed using 
$\gamma \gamma \rightarrow H^{+} H^{-}$are slightly lower than those that can be obtained using $\gamma \gamma \rightarrow l^{+} l^{-}$.

Before we conclude, we would like to mention that the bounds we have presented here are obtained assuming the unpolarized photon beams. The future $\gamma \gamma$ collider can generate either circularly or linearly polarized photon beams. The beam polarization can have some interesting effects on the signal of the NC QED. This analysis is now in progress.

\section{ACKNOWLEDGMENTS}

This work was supported in part by the National Science Council under Grant Nos. NSC 89-2112-M-002-016 and NSC 89-2112-M-002-062, and in part by the Ministry of Education Academic Excellent Project No. 89-N-FA01-14-3.
[1] H. S. Snyder, Phys. Rev. 71, 38 (1947); 72, 68 (1947).

[2] A. Connes, Non-commutative Geometry (Academic, New York, 1994); M. Li and T. Yoneya, Phys. Rev. Lett. 78, 1219 (1997); C.-S. Chu and P.-M. Ho, Nucl. Phys. B550, 151 (1999); A. Connes, M. R. Douglas, and A. Schwarz, J. High Energy Phys. 02, 003 (1998); M. R. Douglas and C. Hull, ibid. 02, 008 (1998); N. Seiberg and E. Witten, ibid. 09, 032 (1999); M. M. Sheikh-Jabbari, Phys. Lett. B 455, 129 (1999); J. L. F. Barbon and E. Rabinovici, ibid. 486, 202 (2000); R. Gopakumar, J. Maldacena, S. Minwalla, and A. Strominger, J. High Energy Phys. 06, 036 (2000); D. Bigatti and L. Susskind, Phys. Rev. D 62, 066004 (2000); N. Seiberg, L. Susskind, and N. Toumbas, J. High Energy Phys. 06, 021 (2000); D. J. Gross, A. Hashimoto, and N. Itzhaki, hep-th/0008075; T. Pengpan and X. Xiong, Phys. Rev. D 63, 085012 (2001); F. J. Petriello, Nucl. Phys. B601, 169 (2001).

[3] Nima Arkani-Hamed, Savas Dimopoulos, and Gia Dvali, Phys. Lett. B 429, 263 (1998); I. Antoniadis, ibid. 246, 377 (1990); I. Antoniadis, C. Muñoz, and M. Quiros, Nucl. Phys. B397, 515 (1993); I. Antoniadis, K. Benakli, and M. Quiros, Phys. Lett. B 331, 313 (1994); I. Antoniadis, N. Arkani-Hamed, S. Dimopoulos, and G. Dvali, ibid. 463, 257 (1998).

[4] C. P. Martin and D. Sanchez-Ruiz, Phys. Rev. Lett. 83, 476 (1999); M. Hayakawa, Phys. Lett. B 478, 394 (2000); hep-th/9912167; Ihab. F. Riad and M. M. Sheikh-Jabbari, J.
High Energy Phys. 08, 045 (2000); M. Chaichian, M. M. Sheikh-Jabbari, and A. Tureanu, Phys. Rev. Lett. 86, 2716 (2001); H. Arfaei and M. H. Yavartanoo, hep-th/0010244.

[5] J. L. Hewett, F. J. Petriello, and T. G. Rizzo, Phys. Rev. D (to be published), hep-ph/0010354.

[6] Prakash Mathews, Phys. Rev. D 63, 075007 (2001).

[7] G. V. Jikia and Yu. F. Pirogov, Phys. Lett. B 283, 135 (1992); L.-Z. Sun and Y.-Y. Lin, Phys. Rev. D 54, 3563 (1996); Surajit Chakrabarti, Debajyoti Choudhury, Rohini M. Godbole, and Biswarup Mukhopadhyaya, Phys. Lett. B 434, 347 (1998); D. K. Ghosh, Prakash Mathews, P. Poulose, and K. Sridhar, J. High Energy Phys. 11, 004 (1999); Prakash Mathews, P. Poulose, and K. Sridhar, Phys. Lett. B 461, 196 (1999); Xiao-Gang He, Phys. Rev. D 60, 115017 (2000); Debajyoti Choudhury and Anindya Datta, Nucl. Phys. B592, 35 (2001).

[8] K. Matsubara, Phys. Lett. B 482, 417 (2000).

[9] Hitoshi Murayama and Michael Peskin, Annu. Rev. Nucl. Part. Sci. 46, 533 (1996); E. Accomando et al., Phys. Rep. 299, 1 (1998).

[10] Ihab. F. Riad and M. M. Sheikh-Jabbari, J. High Energy Phys. 08, 045 (2000).

[11] I. Ginzburg et al., Nucl. Instrum. Methods Phys. Res. 202, 57 (1983).

[12] R. Field, Applications of Perturbative QCD (Addison-Wesley, Reading, MA, 1989). 